\title{
THE ETHICAL DIMENSION OF SYNTHETIC TEACHING OF MATHEMATICS
}

\begin{abstract}
Man has created certain habits and ways of dealing with ordinary life situations. It is possible to talk about regularly repeated patterns of behaviour and of solving the everyday routine problems. In principle, people create algorithms for solutions of individual situations, and these algorithms start for him/her more or less subconsciously in identifying the existing problem. But what happens if he/she meets a new, totally unknown situation where the learned algorithms are not enough? There is an inner conflict of the learned with the unknown.

In our article, we point out the asset of synthetic mathematics teaching when the pupil is led to solve creatively emerging problems using already-identified solutions. Such an educated pupil adds his own code of ethics to solving problems; he/she does not take uncritically offered fast solutions from the surroundings. When searching for a respective solution, there is always an ethical dimension lying in a person expecting from his/her own problems solutions that they will not only be good solutions but also the excellent ones. It is possible to consider his or her own solution that is taken from other people if one understands this solution and accepts it as his/her own.
\end{abstract}

Keywords: code of ethics, synthesis of knowledge, respective solution, problem, the teaching of mathematics

\section{Introduction}

During their lifetime, humans create certain habits and ways of dealing with common life situations. It does not matter whether we are talking about pupils going to school or adults going to work. It is possible to talk about regular repeating patterns of behavior and the solving of everyday routine problems. In essence, one creates algorithms to solve individual situations, and these algorithms are triggered more or less unconsciously when identifying the problem. But what happens if one encounters a new, completely unknown situation where the acquired algorithms are not enough? Such new situations include, for example, joining a new school or starting a job in a new company. It can also be joining a new community of people as well as a holiday journey to an unknown place. But it is much more likely that one encounters an unexpected problem that one has not yet solved in his everyday life. There is an inner conflict of the acquired with the unknown. For a person who encounters a new life situation, there is a need to find a new way of managing it.

We often look for some solutions on the Internet nowadays. But we do not always find a solution to our problem, or the solution offered is not possible in our conditions. We think that school has an exceptional position in the life of a man when meeting something new. In almost every lesson a pupil meets something new, not yet known. It is not only about gaining new knowledge, but also about their organic incorporation into the pupil's mind and the connection with the already acquired knowledge. At the same time, it is necessary to activate this new knowledge, which is realized by their application in solving the problems. Another important aspect of learning new knowledge is to link them to their own code of ethics. There is a need for pupils to lead their knowledge and skills to achieve goals that are beneficial to their surroundings. The way of teaching plays a crucial role in incorporating new knowledge into the mind and heart of pupils.

According to Albert Bandura's research [1], a human can selectively turn on and off their moral principles. At one point they can be barbarously cruel, and a few seconds later they are sensitive and sympathetic. The presence of authority is of great importance for that. In classes, the teacher is an authority for pupils. If a teacher passes on new findings authoritatively and requires the pupils to solve the study problems the same way they always do, the teacher places them in the position of the recipient. Pupils are led to take over the whole problem-solving procedures, the entire algorithms of behavior patterns. This is how they are pushed to turn off their own moral code and take

\footnotetext{
* ${ }^{1}$ Dalibor Gonda, ${ }^{2}$ Marcela Pjatkova

${ }^{1}$ Department of Pedagogical Studies, Faculty of Humanities, University of Zilina, Slovakia

${ }^{2}$ Department of Mathematics, Faculty of Natural Sciences, Constantine the Philosopher University in Nitra, Slovakia

E-mail: daliborgonda@fhv.uniza.sk
} 
over the teacher's. A completely different situation arises when the teacher leads pupils to find solutions to new problems. In this case, the pupils are led to use all their already acquired knowledge and skills in finding a solution to the problem. New knowledge or methods are only offered to them when the task is unresolved with their knowledge. Let us keep in mind that the person who solves the problem independently seeks a solution in accordance with his code of ethics.

There is a great deal of training to solve problems independently for pupils in Math lessons. It was Mathematics that arose as science, whose purpose was to help solve the problems of other disciplines. We think that the way of teaching mathematics has a major influence on the formation of the way of solving the real problems of pupils in their future lives. The way we teach Mathematics, using the knowledge and skills that have already been acquired, is called synthetic teaching. The essence of such teaching of Mathematics is that the pupil is led creatively to solve emerging problems using previously identified solutions. This educated pupils employ their own code of ethics to solve problems, and they do not take uncritically fast solutions from their surroundings. The search for their own solution has always an ethical dimension in that the person expects from their own problem solving that they will not only be good solutions but also beautiful solutions. It is also possible to treat a solution taken from other people as one's own solution if one understands this solution and accepts it as one's own.

\section{Characteristics of the synthetic way of teaching mathematics}

The essence of the synthetic teaching of Mathematics lies in the fact that the pupils deal with appropriate tasks using already acquired knowledge and skills (not only mathematical). New knowledge of Mathematics is presented to them by the teacher at an appropriate time to solve the problem. It is always the knowledge that the student misses to complete the task successfully. In solving the problem, the new knowledge will be integrated into the students' thinking world. The task solving is created by the synthesis of already acquired knowledge and new knowledge if it is needed. From there we deduce the name of the described way of teaching Mathematics. The student, based on the task assignment analysis, identifies the basic knowledge that could be used to solve the task. By identifying the relationships between this knowledge and their interconnection or combination, they will gradually create a solution to the task.

The great benefit of the synthetic teaching process is the expansion of students' solving skills. They realize that it is not always necessary to learn new knowledge and new ways of solving new problems. They will learn how to solve new tasks first by using what they already know and complete new findings only when needed. On the one hand, the student's solving self- confidence is strengthened and, on the other hand, the process of lifelong education will start off. Solving their self-confidence will support solving their enthusiasm (they will begin to solve the problem), and the natural desire to know the result inspires them to complete their knowledge if it is necessary to solve the task. After incorporating the new knowledge into the thinking world of pupils while solving the task, it is necessary to create an organic link to new knowledge with the knowledge already acquired. This is carried out by a wide range of various examples where the new knowledge is used in different forms and contexts. The synthesis is completed only when the pupil acquires new knowledge at the application level - he/she can creatively handle it in solving the problems and the form of its use is in accordance with his code of ethics. In the pupil's mind, a new world of knowledge is created, which is manifested by the increased ability to solve the problems. When applying a synthetic way of teaching Mathematics, the choice of tasks is the key. The teacher should choose the tasks in which the learner can solve a few initial steps independently. At a certain stage of the solution, they encounter the problem of their lacking knowledge and the teacher's help is needed. After acquiring the missing knowledge, they continue solving the task independently. The second key type of tasks are those that the students can solve independently. After solving them, the teacher presents them a more effective method of solving a given task.

The solution of the task in a synthetic way has the following steps:

1. Activation of the necessary knowledge and skills based on the task assignment analysis

2. Step-by-step creation of a task solution

3. Conflict - question their own ability to solve the task

4. Addition of missing knowledge

5. Completing the task based on their own knowledge

We repeat that after solving the problem where the pupils were enriched with new knowledge, it is necessary to include various tasks in which the solution will use a new piece of knowledge or method of solution. When choosing the tasks, we are not limited to one thematic unit, but we select tasks from different areas of Mathematics. The advantages of synthetic teaching are presented on the example of analytical geometry, which itself came from the synthesis of other parts of Mathematics.

\section{Analytical geometry}

Analytical geometry is an area of Mathematics in which geometric objects are studied through their analytical expressions. French mathematicians, Rene Descartes (1596-1650) and Piere De Fermat (1601-1665), are considered the founders of analytical geometry. The emergence of the unity of algebra and geometry led to the emergence of this new area of Mathematics. The essence of the analytical solution of a geometric task is to convey the task by means of coordinates to the algebraic task, usually 
to solve equations, inequations, and their systems [2]. With the chosen coordinate system we can express each basic geometric figure uniquely in the form of a certain equation (or inequation). The relationship between the respective geometric form and its equation is given by the following rule:

Any point $X$ lies in a given object if its coordinates match the equation of the object.

Based on this rule, the penetration of the two formations is the set of all points, the coordinates of which correspond simultaneously to the equations of the two units [3].

The basic idea of the emergence of analytical geometry - the discovery of new knowledge and the possibilities of solving problems by utilizing the interconnection of several areas of Mathematics, can become an inspiring element for the development of mathematical thinking of pupils and the teaching of Mathematics. During Math classes, pupils acquire new knowledge from Mathematics and acquire solving skills. New findings are presented to them by the teacher in a certain mathematical environment, which most often forms a thematic learning unit. Teaching is mostly subordinated to a given thematic unit. The study of mathematical concepts, as well as the selection of examples and tasks, are framed within a given thematic unit. Such mathematical teaching scenario often leads to the emergence of isolated knowledge in the minds of pupils [4]. The content of mathematical concepts and methods of solution, however, are not limited to the thematic areas of Mathematics but often have an "all-mathematical" scope.

The primary goal of teaching Mathematics is to develop pupils' thinking, and in pursuit of this goal, it is necessary to develop the ability to use creatively the knowledge to solve the tasks of everyday life. Thus, the teaching of Mathematics is beyond the limits of Mathematics itself. We think that the way to overcome the isolation of individual knowledge and the teaching of Mathematics from everyday life has been suggested by the creators of analytical geometry. The synthesized method of teaching Mathematics that we describe is inspired by the emergence of analytical geometry. The basic idea is to make the most of the knowledge and skills that have already been acquired in creating the solution of the task. The need for new knowledge arises in the process of solving the task as a natural element of cognition and problem-solving. Such approach to problem-solving has a significant impact on the future way of solving the problems that people meet in their everyday life. The pupil is prepared for the role of the researcher, is led to use one's own abilities creatively. At the same time, they are aware of their own borders and the need for cooperation with the environment, which also enhances one's ethical behavior. For the successful application of the synthetic teaching method, it is necessary to gradually create a good mathematical basis - a portfolio of theoretical knowledge acquired at least at the level of understanding. The individual elements of the portfolio are interconnected in one organic unit on the basis of suitably chosen examples and tasks. Students will gradually realize that all tasks are solved by a suitable combination and application of theoretical knowledge. At the same time, they gradually acquire the basic methods of solving the problems. The students will solve most of the tasks individually or with little help from the teacher. The awareness of one's own ability to solve a task is an insignificant motivating factor to advance in the acquisition of mathematical knowledge.

\section{Possibilities of using the synthetic teaching of analytical geometry}

As we mentioned above, analytical geometry arose as a synthesis of two areas of Mathematics. It could be said that we think geometrically when solving a problem and find the solution algebraically. by looking at the usual analytical geometry textbooks, however, we find that individual knowledge of analytical geometry is presented in them in a relatively separate and simplified form. Geometric reasoning is used predominantly to create a formula that is subsequently used as a substitution for geometric reasoning. In task collections, the emphasis is placed on practising the solution of tasks based on the use of formulas. In essence, basic algorithms are practised, with a series of formulas in the center. In this way, pupils can create an image of analytical geometry as a relatively separate part of Mathematics. Analytical geometry becomes a set of formulas for them to learn how to use them in solving a task. The success of problem-solving in analytical geometry is conditioned by memorizing a large number of formulas. According to research, such approach leads to $\mathrm{s}$ passive acquisition of knowledge by students and often to their resignation [5]. Malcolm Gladwell in his Outliers, writes that ten thousands of hours of practice are needed to master a subject field [6]. If we transfer this idea to teaching Mathematics, students will not be mathematically mature due to the number of hours spent by calculating mathematical tasks, often of the same type (just changing numbers). Mathematics requires many hours of mathematical work to master it. It consists of solving a variety of tasks requiring conceptual thinking. Through this way of thinking, pupils learn how to choose, use and apply different methods in solving tasks. In essence, they form their own task solving based on their knowledge and skills. They do not use finished products offered by a teacher or a textbook, but they make their own and often original solutions. In this context, another problem with textbooks is that tasks are largely taken out of the context, only mathematical tasks are solved. The pupils then do not know where the knowledge can be applied, which significantly reduces their motivation to learn [7]. We think that the teaching of analytical geometry, having mathematical formulas in its center, results in the isolation of this thematic unit from the other parts of Mathematics. This way of teaching has little impact on the development of independent creative thinking. At the same time, it may also have overlap into their ethical behavior. In the case of 
life-threatening conflicts, they can replace their own way of solving by searching for a quick solution - some recommended behavioral pattern. Recommended behavioral patterns help humans, but they do not always comply with their code of ethics. Conflict occurs most often when two (or more) heterogeneous elements collide. This can happen within a thinking subject - internal conflict, or in relation to other people - external conflict. However, conflict does not necessarily lead to something negative. Conflict has energy that can be used to find new valuable solutions. TThis successful "meeting“ of two parts of Mathematics can be illustrated by an example of searching for a tangent to the circle. There is also a symbolic subtone for selecting a sample example as for tangent we look for common points that occur at the center of two geometric formations.

\section{Tangent to the circle}

In common textbooks, the study of the tangent to the circle begins by deducting the general equation of the tangent to the circle. However, pupils are often offered, especially for reasons of time, the given equation as a finished product. This is followed by examples and tasks for using this equation. However, it is not necessary to know the general equation of the tangent to solve the problems of the tangent. This equation can be discovered when solving tasks in an analytical geometry environment. To discover this equation, the knowledge about the circle and line from planimetry and knowledge from algebra is sufficient. Thus, it is possible to solve the problem in the spirit of the founders of analytical geometry. We will solve this task by synthesizing knowledge mainly from these two parts of Mathematics. In the spirit of the synthetic way of teaching, pupils will be enriched with new concepts of analytical geometry during the solution. In other words, the conceptual apparatus of analytical geometry will be constructed during the task solving.

Example: This is the equation of the circle. $k:(x-3)^{3}+(y+12)^{2}=100$. Find the general equation of the tangent to the circle $k$ that passes through points a) $L[9 ;-4]$, b) $M[5,2]$.

\section{Solution:}

a) The point $L$ may be inside the circle, on the circle, or outside of the circle. Depending on its position, the way the task is solved is determined. From planimetry we know that the tangent can be constructed only in the second two cases of the position of the point $L$. To determine its position, it is enough to compare its distance from the center of the circle with its radius. We position the point $L$ with respect to the circle by fitting the coordinates of the point $L$ into the circle equation $k$. After fitting $L S=P S$, it means that the point $L$ is a circle $k$ and is the touch point of the tangent.

The searched tangent is a straight line, thus it will have a general equation $t: a x+b y+c=0$. To determine the parameters $a, b, c$, we will use the knowledge of the planimetry. The radius of the circle constructed at the touch point is perpendicular to the tangent at that point. This means that the vector $\overrightarrow{S L}$ ( $S$ the center of the circle $k$ ) is a normal vector of the tangent $t$, its coordinates $\overrightarrow{S L}=(3 ; 4)$ are the searched parameters $a, b$. To determine the value of the parameter $\mathrm{c}$, we use the point $L$ that fits the tangent, and after the coordinate point $L$ is inserted into the equation $3 x+4 y+c=0$, we get the solution $3 x+4 y+c=0$. The searched tangent to the circle $k$ has the equation $t: 3 x+4 y-11=0$.

b) Like the solution a), first we determine the position of the point with respect to the circle. Its distance from the center of the circle $S$ is $2 \sqrt{32} \doteq 12,2$, which is more than the radius of the circle. The point $M$ is outside the circle and it is possible to construct two tangents to the circle $k$. For this reason, there will be two general equations of the tangents. Even in solution b) we will proceed by using the knowledge of planimetry. We know that if we want to build a tangent to a circle we need to know the touch point. This would be constructively build with a Thales' circle $k_{T}$ with a center at the center of the line $S M$ and a radius $r_{T}=\frac{1}{2}|S, M|$. Therefore, as the first step of the solution, we find the analytical expression of the Thales' circle $k_{T}\left(O, r_{T}\right)$. The point $O$ is the center of the line $S M$, so it has the coordinates $O[4 ;-5]$ and the radius is $r_{T}=5 \sqrt{2}$. On the basis of the obtained values, we draw the equation of the Thales' circle. $k_{T}:(x-4)^{2}+(y+5)^{2}=50$

Touchpoints are common points of circles $k$ a $k_{T}$. In the case of the analytical representation of the circles, this means that the coordinates of the touch points are the solution of the following system

$(x-3)^{2}+(y+12)^{2}=100$

$(x-4)^{2}+(y+5)^{2}=50$

We will use the knowledge of algebra to address it. After adjusting the individual equations, we get the system in in the form

$x^{2}+y^{2}-6 x+24 y+53=0$

$x^{2}+y^{2}-8 x+10 y-9=0$

After deducting equations and adjusting, we get the equation $x+7 y+31=0$

From the algebra perspective, this is an equation with two unknowns that do not have a definite solution. However, the given equation can be perceived as a new condition that must be met by the coordinates of the intersections of the given circles and thus the coordinates of the searched touch points. If we look at this equation with the "eyes" of analytical geometry, we have a general straight line before us. The finding that the intersections of the circles lie on the line is not surprising since every two points clearly determine the straight line. However, we know from the planimetry that the intersections of the two circles lie on the line perpendicular to the centerline of the circles. The 
perpendicularity of the line $p: x+7 y+31=0$ to the line $\overrightarrow{S O}$ is shown by the fact that the vector $\overrightarrow{S O}=(1,7)$ is the normal vector of the line $p$. Based on the above, we can say that the coordinates of the searched touch points must match the analytical expression of circles, $k_{T}$ and lines $p$. As the next step, we compile a simpler system of equations for calculation

$$
\begin{aligned}
& (x-3)^{2}+(y+12)^{2}=100 \\
& x+7 y+31=0
\end{aligned}
$$

We will solve this system by a substitution method. Its solution consists of two arranged pairs, which are at the same time the coordinates of the touch points of the tangents from the point $M$ to the circle $k$. The touchpoints have coordinates

$$
T_{1}[-3,-4] \text { a } T_{2}[11,-6]
$$

After construction of the touch points, it is possible to construct tangents from a planimetry point of view. Even with the analytical solution, it is already possible to write the equation of the tangents, because each of them already knows two different points. The tangent $t_{1}$ is determined by the points $M, T_{1}$ and the tangent $t_{2}$ is determined by the points $M, T_{2}$. The formation of general equations of the tangents is a basic skill in analytical geometry. Searched tangents have equations

$$
t_{1}:-3 x+4 y+7=0 \text { a } t_{2}: 4 x+3 y-26=0
$$

\section{Evaluation of the task solving procedure and topics for discussion}

In the above-mentioned way of solving the task, we have consistently followed the basic idea of creating analytic geometry. When creating a task solution, we used the same idea as if it were a planimetric task designed to construct a tangent to a circle. The only change is that we used algebra elements to implement the individual steps of the solution. In other words, the algebraic tools - the equation - were used instead of geometric tools. In this sense, the solution offers pupils an alternative - geometric reasoning can be written using algebra, so that the position of individual points can be determined by means of coordinates. Such an approach is advantageous, for example, in determining the positioning of geometric configurations in stereometry for pupils who have a spatial imaging problem. A significant benefit is developing the ability to perceive equations as real-world geometric objects. The entry $(x-3)^{2}+(y+12)^{2}=100$ is no longer perceived as an equation (algebraically) but also as a circle (geometrically). As a circle that is not drawn but written. As we can see from this picture and from this entry, where is its center and what its radius is. In this way, the pupils also acquire the necessary mathematical super vision that goes beyond both geometry and algebra. This is also manifested in the solution of the system of equations, where they are constantly aware that while solving the system of equations, the result will be common points of the circles. From their experience of system solving they know that after equations subtraction $x^{2}+y^{2}-6 x+24 y+53=0$

$x^{2}+y^{2}-8 x+10 y-9=0$

they will get an "insoluble" equation from the point of view of algebra. But a geometric view says that by subtraction we get the straight line equation. This conflict is solved very smartly. What makes algebra no more insoluble is a valuable inter-result in terms of geometry that will be useful in finding the final solution. The location of the search for the continuation of the system solution, or another way of solution, is followed by a geometric survey of the properties of the obtained line. Based on the research of the new object - lines, we have gained new insights. The equation that is derived by subtracting the general equations of the circles is the equation of the line perpendicular to the centerline of the circles, and the intersections of the given circles are located on them. Therefore, the searched intersections of the circles can be obtained as intersections of one of the circles with the obtained line. Thus, it was possible to construct a new, simpler system of equations

$$
\begin{aligned}
& (x-3)^{2}+(y+12)^{2}=100 \\
& x+7 y+31=0
\end{aligned}
$$

After solving the problem, the solution described above offers a space for natural discussion. Firstly, the geometric debate. The question arises whether by subtracting the two general equations of the circles, we obtain a line on which there are intersections of the circles. By using simple examples, it can be shown that by subtracting the two general equations of the circles we always get the straight line equation. It is always perpendicular to the centerline. But it can not be used to effectively search for the mutual position of circles because we would have to find its intersections with both circles separately. However, if we know that the circles are intersecting, its use leads to a more efficient way of sharing their points. The geometric and algebraic view is constantly overlapping with the solution. Primary is geometric since it is a geometric task. Algebra serves as an effective tool for realizing geometric ideas and solution steps. At the heart of pupils' reflections are not equations but geometric objects - circles and lines. The results of their findings are put down symbolically (algebraically).

We can also lead an algebraic discussions. Pupils are taught that, when solving a system of equations with two unknowns by an addition methods, they try to create one equation with one unknown, which they then solve and the result fits into one of the original equations to get the value of the other unknown. In essence, they have learned that by using the addition method, after the addition of equations, one of the original equations can be used only if one already knows the value of one unknown. In the example solution mentioned, the two "rules" were violated. This has led us to the object perception of equations and to the geometric interpretation of the solution of the system of equations. Pupils are enriched by the knowledge that by adding together two equations we have acquired not only the new equation but also the new condition that must be met by the sought solutions of the 
system of equations. The new condition means the new equation that the search points must meet. Its object interpretation and determination of its relationship to the objects examined will reveal the possibilities of its use in solving the task. They realize that the result of solving the system of equations may be not only single points but also a whole line. Pupils gradually perceive that mathematical equations receive content to solve real problems. It is possible to say that the equations come to life in real conditions and are ready to communicate with the solver and gradually reveal their secrets. Knowing that the equations are realistically interpretable has never pushed humanity forward.

\section{Conclusion}

The proposed teacher's code of ethics also includes the following point: "Teacher leads their pupils to independence and critical thinking. Within their and also their pupils' abilities and possibilities, they strive for the complete development of the pupil personality" [8]. In this context we consider the following essential to keep in mind: that Mathematics has arisen to help people solve problems. Teaching Mathematics has a legitimate ambition, to be the enrichment of man's solving abilities. And in this spirit, there is still an up-to-date question of finding new methods of teaching Mathematics. The problem of students understanding and mastering Mathematics is currently one of the most emphasized topics of expert public discussion. New ways of teaching mathematics more effectively and attractively are being searched for [9].

In general, a common feature of new methods is that the pupil's personality and not the Mathematics itself is the center of learning. Mathematics should be incorporated into the pupil's thinking and gradually enriched. During the process of teaching, one (mathematical) problem needs to be handled at different levels of complexity, one more variation in one situation. There is a need to guide pupils to find creative and often unusual ways to solve the problem. We think that the synthetic way of teaching Mathematics is one of the possible ways of a complex development of a pupil's personality. It leaves the student with the solving freedom and supports their critical thinking. The pupil is not in the position of the receiver of the complete problem solving but is led to create their own solutions to the problems encountered. If a pupil is taught separately during schooling and thinking, there is a great assumption that later in life they will critically accept the opinions offered and solve the problems. They will be "set" to accept only solutions that do not conflict with their own code of ethics. If such a solution is not offered to them, they will come up with their own, ethically "clean" solution, making themselves a contribution to their surroundings.

\section{References}

[1] OSOFSKY, M. J., BANDURA, A., ZIMBARDO, P. G.: The Role of Moral Disengagement in the Execution Process. Law and Human Behavior, 29(4), 371-393, 2005.

[2] ZNAM, S., et al.: View into the History of Mathematics (in Slovak). Alfa, Bratislava, 1986.

[3] POLAK, J.: An Overview of the Secondary-School Mathematics (in Czech). Prometheus, Praha, 2003.

[4] HEJNY, M.: Teaching Mathematics Focused on Schemes Building: Arithmetic 1st Level (in Czech). Univerzita Karlova v Praze, Prague, 2014.

[5] BOALER, J., GREENO, J.: Identity, Agency and Knowing in Mathematics Worlds. Multiple Perspectives on Mathematics Teaching and Learning. Westport, CT, Ablex Publishing, 171-200, 2000.

[6] GLADWELL, M.: Outliers: The Story of Success. Back Bay Books, Hachette Book Group, New York, 2011.

[7] BOALER, J. : Mathematical Feeling (in Slovak). Tatran, Bratislava, 2016.

[8] PAJTINKA, L: Teachers Belong to Heaven (in Slovak). IS-SET, 2008.

[9] KONTROVA, L. LENGYELFALUSY, T., LENGYELFALUSYOVA, D.: A Statistical Analysis of the Effectiveness of Selected Methods in the Teaching of Mathematics. Communications - Scientific Letters of the Univesity of Zilina, 14(1), 55-60, 2012. 